\title{
The VLT Interferometer
}

\author{
A. Richichi \\ European Southern Observatory, \\ Karl-Schwarzschildstr. 2, 85748 Garching b. M., Germany \\ email: arichich@eso.org
}

\begin{abstract}
The ESO Very Large Telescope Interferometer (VLTI) is arguably the most powerful optical interferometric facility available at present. In addition to the wide choice of baselines and the light collecting power of its $8.2 \mathrm{~m}$ and $1.8 \mathrm{~m}$ telescopes, the VLTI also offers a smooth and user-friendly operation which makes interferometry accessible to any astronomer and covers a wide range of scientific applications. Behind the routine scientific operations, however, the VLTI is in constant evolution. I will present some of the technological and instrumental improvements which are planned for the near and mid-term future, and discuss their implications for astrometry in particular. Among them, the PRIMA facility and the proposed GRAVITY instrument are designed to reach the level of 10 microarcseconds in the near-infrared.
\end{abstract}

Keywords. instrumentation: high angular resolution, techniques: interferometric, astrometry

\section{An introduction to the VLTI}

Cerro Paranal in the chilean Atacama desert is a world-renowned astronomical site. It is home to four $8.2 \mathrm{~m}$ so-called Unit Telescopes (UTs), and to four $1.8 \mathrm{~m}$ Auxiliary Telescopes (ATs), as well as to other telescopes. The UTs and the ATs can be combined interferometrically by means of subterranean light ducts and delay lines. The maximum baselines that can be realized by combining UTs or ATs are about $130 \mathrm{~m}$ and $205 \mathrm{~m}$, respectively, with a large number of different configurations in terms of baseline length and orientation (Fig. 1). In particular, the ATs can be moved over 30 stations, and their relocation can be accomplished in a few hours (Fig. 2).

The VLTI (Glindemann et al. 2003) had first fringes in March 2001. For about three years, the test instrument VINCI (Kervella et al. 2000) was used to commission the VLTI. The result is a public database of about 20,000 observations on several hundred of stars. A significant fraction of these data have been used, mainly by researchers outside ESO, to obtain impressive new scientific results, published in tens of refereed papers. By the end of 2002 and early 2004, respectively, the two facility instruments MIDI and AMBER arrived on Paranal. They were subsequently commissioned and made available to the community along the same guidelines as thesingle telescope instruments.

MIDI (Leinert 2004) is a 2-way beam combiner with spectral resolution up to $\lambda / \Delta \lambda=$ 230 in the $\mathrm{N}$ band. It achieves a sensitivity of few tenths of Jy at the UTs, in self-fringe tracking mode. Better sensitivities are possible, mainly with the off-axis fringe-tracking capability of PRIMA discussed later. The combination of an mid-infrared constructive (i.e. not nulling) beam-combiner with the apertures and baselines of the VLTI is unique in the world, and a large number of the papers resulting from MIDI are indeed first time results in their own area.

AMBER (Petrov et al. 2007) can combine up to 3 beams at wavelengths between 1 and $2.5 \mu \mathrm{m}$, with spectral resolution up to $\lambda / \Delta \lambda=10^{4}$. Although other near-IR beam combiners exist in the world with the capability of realizing three or more baselines, AMBER at the VLTI is unique in the simultaneous use of large telescopes and long 


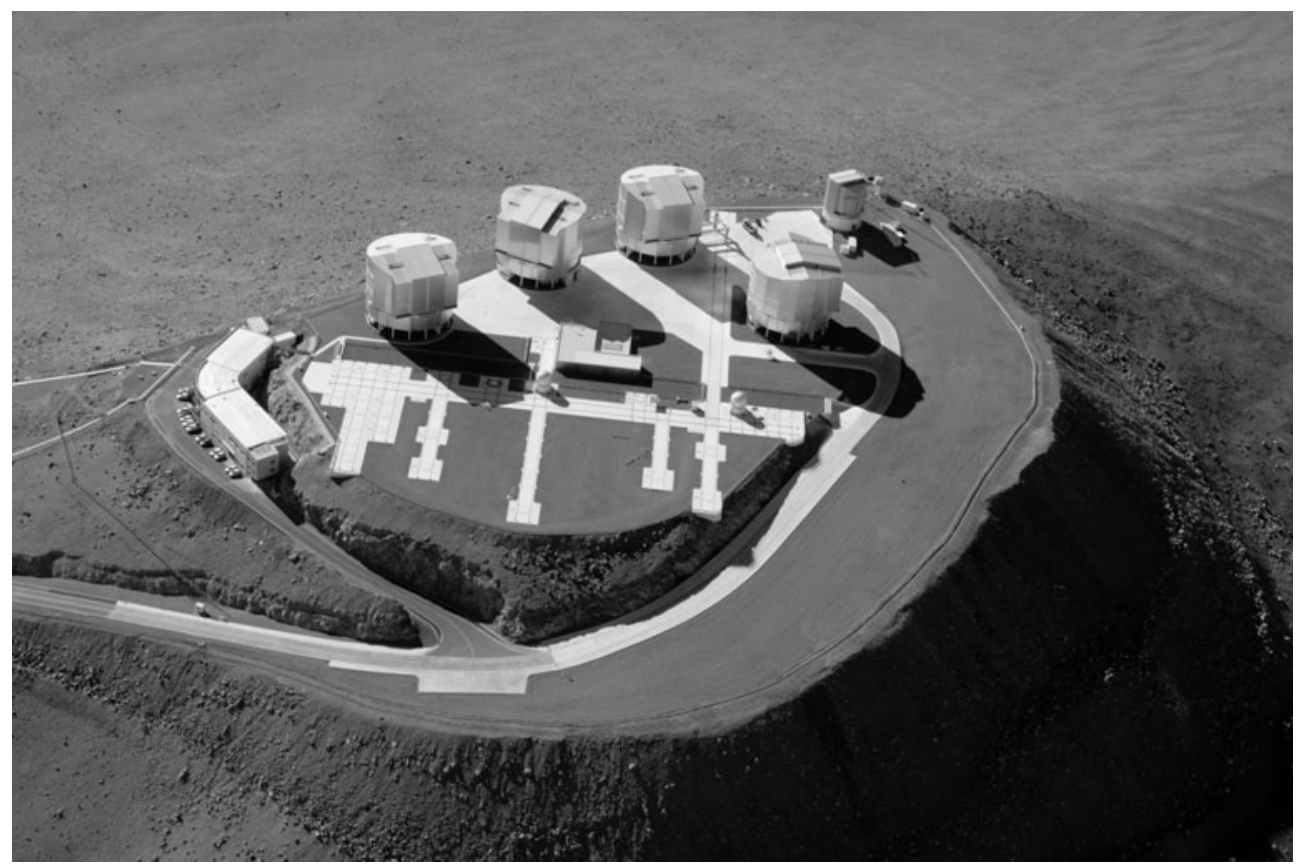

Figure 1. Aerial view of the Paranal site. In addition to the four $8.2 \mathrm{~m}$ Unit Telescopes, also two Auxiliary Telescopes (total of four by now) are visible. The ATs can be moved over a grid of 30 stations by rails. Observations are carried out from the Control Building on the left of the photo. All these telescopes can be combined interferometrically, with the exception of VST at the top right.

baselines. Results from AMBER are starting to be published at an encouraging rate, spanning a wide range of scientific topics.

Next to MIDI and AMBER, the VLTI includes a large number of complex subsystems, including adaptive optics on the UTs (MACAO, Arsenault al 2003), a visible tip-tilt corrector for the ATs (STRAP, Bonaccini al 1997), six delay lines (Hogenhuis et al. 2003), an H-band fringe tracker (FINITO, Gai et al. 2004), an IR tip-tilt corrector in the underground laboratory (IRIS, Gitton et al. 2004), a reference source (ARAL, Morel et al. 2004). The lab itself, now quite crowded, is schematically shown in Fig. 3.

ESO has conceived the VLTI to be not only a powerful interferometric facility, but also as one open and accessible to the general astronomical community. It is a fact that the majority of, if not all, interferometers are operated and used mostly by the same persons that designed and built them. Observations are often complex, the data demand local specialized software, and their analysis often requires an in-depth knowledge of the peculiarities of the interferometer used. At the VLTI however, most of these complications do not apply. Astronomers can request time, create the instructions for their observations and have them executed in service mode if so wished, and receive their data in a standard format as for any other single-telescope instrument at Paranal. Data are quality-checked and archived, and automated data reduction pipelines are run routinely.

Although the implementation of interferometry as a user-friendly technique has required a large effort at ESO, the outcome is certainly impressive. Each semester, about one hundred proposals are submitted and a large fraction of these are executed in service mode on MIDI and AMBER. Since the opening of the VLTI to the community, over 300 different programs have been or are about to be executed, with 116 different PIs from 15 


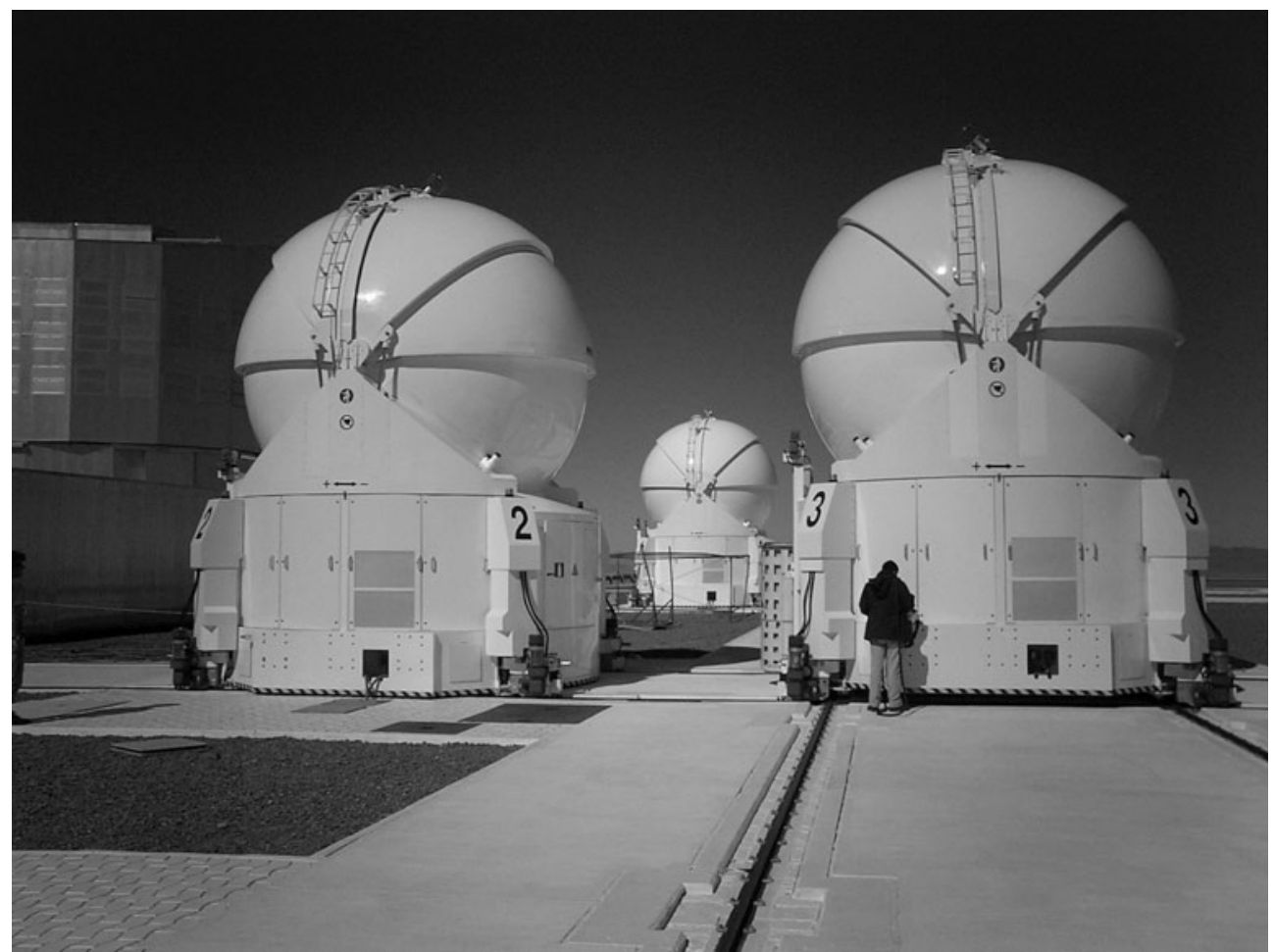

Figure 2. A view of thee of the four Auxiliary Telescopes present on Paranal.



Figure 3. Scheme of the VLTI underground laboratory and delay lines, and of the main instruments and subsystems.

countries. It is important to stress that the VLTI is accessible not just to the researchers in the ESO member states, but to anyone in the world. 


\section{The future of astrometry at the VLTI}

The VLTI is a fully functional facility with a complete set of instruments, but developments are under continuous evolution in order to maintain its leading status in interferometry throughout the next decade. Plans have started long ago to implement a dual-feed facility (PRIMA, Delplancke et al. 2006). This allows to perform interferometry, simultaneously and independently, on two targets separated by up to 1 arcminute. There are several uses of this mode: for example fringe tracking on a relatively bright nearby source, and long integrations (thus much fainter magnitudes) on a faint science target. Or, using the phase information between the two stars (one of which is supposedly with a known, fixed phase), to reconstruct images of the science target. Finally, and perhaps most interestingly for the purpose of this conference, there is the astrometric mode of PRIMA, in which minute changes of angular distance between the reference and the science target can be detected, and monitored in time. Accuracies of $50 \mu$ as are foreseen initially, up to $10 \mu$ as at a later stage when a precise models of the geometry of the telescope is built from many accumulated observations. This would allow, among other things, the direct detection of exoplanets. A consortium has been formed, to develop specific hardware, the so-called Differential Delay Lines, and software to this goal (see Launhardt in these proceedings).

PRIMA is largely built, and it will be deployed in Paranal in 2008. An intensive period of commissioning will follow, with the goal of first science observations later in the same year or early 2009.

Another step towards precise astrometry is GRAVITY (Gillessen et al. 2006), one of the second generation VLTI instruments which have recently completed their Phase A studies and have been recommended for further development. GRAVITY is designed specifically to achieve $10 \mu$ as astrometric accuracy on the faint infrared stars around the supermassive black hole at the center of our own Galaxy (see Gillessen in these proceedings). In addition to a direct measure of the black hole mass, it will be used to detect and investigate a number of strong gravitational effects, effectively serving as a unique physics laboratory. Of course, the high astrometric accuracy of GRAVITY can also be applied to a number of other astrophysical topics. The deployment of GRAVITY at the VLTI is foreseen around 2013.

\section{References}

Arsenault, R., Alonso, J., Bonnet, H. et al. 2003, in SPIE Proceedings, vol. 4839, p. 174

Bonaccini, D., Gallieni, D., Biasi, R. et al. 1997, in SPIE Proceedings, vol. 3126, p. 580

Glindemann, A., Algomedo, J., Amestica, R. et al. 2003, in SPIE Proceedings, vol. 4838, p. 89

Delplancke, F., Derie, F., Leveque, S. et al. 2006, in SPIE Proceedings, vol. 6268, p. 27

Gai, M., Menardi, S., Cesare, S. et al. 2004, in SPIE Proceedings, vol. 5491, p. 528

Gillessen, S., Perrin, G., Brandner, W. et al. 2006, in SPIE Proceedings, vol. 6268, p. 33

Gitton, P., Leveque, S., Avila, G., Phan Duc, T. 2004, in SPIE Proceedings, vol. 5491, p. 944

Hogenhuis, H., Visser, M., Derie, F. 2003, in SPIE Proceedings, vol. 4838, p. 1148

Kervella, P., Coude de Foresto, V., Glindemann, A. et al. 2000, in SPIE Proceedings, vol. 4006, p. 31

Leinert, C. 2004, in SPIE Proceedings, vol. 5491, p. 19

Morel, S., Vannier, M., Menardi, S. et al. 2004, in SPIE Proceedings, vol. 5491, p. 1079

Petrov, R., Malbet, F., Weigelt, G. et al. 2007 A\&A, 464, 1 